\title{
Intergenerational Transmission of Depression: Test of an Interpersonal Stress Model in a Community Sample
}

\author{
Constance Hammen and Josephine H. Shih \\ University of California, Los Angeles
}

\author{
Patricia A. Brennan \\ Emory University
}

\begin{abstract}
An interpersonal stress model of depression transmission was tested in a community sample of nearly 800 depressed and never-depressed women and their 15-year-old children. It was hypothesized that maternal depression (and depression in the maternal grandmother) contributed to chronic interpersonal stress in the mothers, affecting quality of parenting and youths' social competence. In turn, poor social functioning and interpersonal life events caused at least in part by the youths were predicted to be the proximal predictors of current depressive symptoms and diagnoses. Structural equation modeling confirmed the predicted associations among variables and the link between youth chronic and episodic interpersonal stress and depression. Additionally, the association between maternal and child depression was entirely mediated by the predicted family and interpersonal stress effects.
\end{abstract}

Depression in adolescents is relatively common, but it is impairing and may portend recurrent depressive episodes in both community and clinical samples (Lewinsohn, Rohde, Klein, \& Seeley, 1999; Pine, Cohen, Gurley, Brook, \& Ma, 1998; Rao, Hammen, \& Daley, 1999; Weissman et al., 1999). Youth depression undoubtedly arises from various, and typically multiple, causes in different adolescents. However, one risk factor that is believed to be among the strongest predictors of depression in childhood and adolescence is maternal depression (see reviews by Beardslee, Versage, \& Gladstone, 1998; Downey \& Coyne, 1990; Gelfand \& Teti, 1990).

Despite considerable research demonstrating the effects of maternal depression on youngsters' risk for depression, important gaps in the field remain. One major issue is the question of whether the risk to youths results mainly from maternal depression as such, or whether the youths' depression outcomes are largely due to the mediating or moderating effects of adverse conditions that are commonly associated with maternal depression. For instance, depression in mothers typically occurs in the context of marital discord, paternal psychopathology, stressful economic and work difficulties, and negative interactions with the children (see reviews by Cummings \& Davies, 1994; Downey \& Coyne, 1990; Goodman \& Gotlib, 1999; Hammen, 1991; Lovejoy, Graczyk,

Constance Hammen and Josephine H. Shih, Department of Psychology, University of California, Los Angeles; Patricia A. Brennan, Department of Psychology, Emory University.

This research was supported by National Institute of Mental Health Grant R01MH5223901. We thank Jodie Ullman and Kevin Kim for statistical consultation. We are grateful to the project staff in Brisbane, Robyne Le Brocque, and interviewers Cherie Dalton, Barbara Mann, Eileen Tone, Sandra Fergusson, Lisa Manning, and Molly Robbins, as well as to Jake Najman, William Bor, Michael O'Callaghan, Gail Williams, and Margaret Andersen of the University of Queensland.

Correspondence concerning this article should be addressed to Constance Hammen, Department of Psychology, University of California, Los Angeles, CA 90095. E-mail: hammen@psych.ucla.edu
O'Hare, \& Neuman, 2000). All of these variables have themselves been shown to predict children's symptoms and behavioral difficulties. The question is whether maternal depression may have a direct effect on youth depression-presumably via genetic or psychological processes-or whether the effects are mostly accounted for by the stressors in the child's life and maladaptive learning experiences that accompany parental depression.

A related gap in the field concerns the relative paucity of studies that have clarified risk mechanisms and the potentially complex associations among variables thought to contribute to children's negative outcomes. Goodman and Gotlib (1999), for example, examined in detail the multiple factors likely involved in children's risk because of maternal depression and have called for testing complex models as a way of addressing the unresolved questions. A few studies have examined multiple risk factors, and their results provide useful clues about the role of various contextual factors but inconsistent evidence of the direct effects of parental depression itself (e.g., Billings \& Moos, 1985; Fergusson, Horwood, \& Lynskey, 1995; Goodman, Brogan, Lynch, \& Fielding, 1993; Hammen, Burge, \& Stansbury, 1990; Seifer et al., 1996; Warner, Mufson, \& Weissman, 1995). Moreover, these studies have been limited in the number of risk factors included and in the complexity of the predictive models tested.

There are several reasons why the studies of mechanisms of risk have not extensively tested complex models. Most studies have been based on clinical samples with parents whose disorders may be severe and represent unusually adverse contexts-conditions that may not generalize to the vast majority of depressions experienced in community populations. Also, for the most part, previous studies have not been large enough, have not collected multiple variables to begin to examine the processes of risk to children, or have not chosen to explore the mechanisms of risk. The current study is a large community-based sample with multiple variables, providing a basis for beginning to test complex models of risk processes predictive of adolescent depression. Variables included in the present analyses were based on interview and self-report 
methods, and where feasible, were based on multiple methods of assessment.

The present study is a test of an intergenerational interpersonal stress model of depression in young adolescents. It is based on a community sample of 816 families selected for varying histories of maternal unipolar depression or no depression. Many of the mothers had current or past diagnoses of major depressive episode (MDE) or dysthymic disorder, many with multiple episodes. The families were studied in depth when the target child was 15 years of age, and information was obtained about the diagnostic status of the youth, mother, father, and mother's parents, mother's recent stressors, family relationships including marital and parent-child functioning in the prior 6 months, and youth social functioning and recent episodic stressors. The present cross-sectional study focuses primarily on youths' current depression in order to make the best possible case for the effects of predictors operating prior to the current symptoms.

The intergenerational interpersonal stress model of depression was first proposed by Hammen (1991a) in a sample of 8-16-yearold children of mothers who were clinically ascertained to be depressed; families with unipolar, bipolar, medically ill, and well mothers were compared. Hammen (2002) further elaborated the role played by stressful conditions in families of depressed parents, including family, marital, and parenting stress, as well as the role of depressed parents' modeling of inadequate social problemsolving skills and poor coping with social stressors. The model proposes that the negative effects of maternal depression on youths' depressive outcomes are largely due to the stressful family environment and its consequences for youths' interpersonal functioning. Such environments commonly include a father with psychopathology, as well as the youth's exposure to poor marital relationships and to evidence of the mother's interpersonal difficulties with others in her social world, such as her extended family and friends (e.g., Hammen \& Brennan, 2002). The youths may directly observe relatively maladaptive ways in which to relate to others and witness poor skills for preventing or resolving interpersonal disputes. Such learning experiences potentially create a relative lack of social competence and contribute to the generation of stressful interpersonal life events (e.g., Adrian \& Hammen, 1993; Hammen, 1991b). Because stress is commonly the immediate trigger of depressive experiences (e.g., Brown \& Harris, 1989), relatively higher levels of youth chronic and episodic social stress will likely predict depressive reactions, especially among youths at risk.

The model also considers that maladaptive parenting may be an important quality of the family life of depressed mothers, stemming largely from highly stressful family environments that interfere with optimal parenting (see review in Goodman \& Gotlib, 1999). Parenting quality, especially if perceived as being negative by the child, is itself stressful, and it contributes, through maladaptive learning, to less social competence and to stressful interpersonal experiences.

There are several key ingredients and predictions of the model, as illustrated in Figure 1:

1. The model is intergenerational in two senses: Maternal depression is a key element of prediction of youth depression, hypothesized to have both direct and mediated effects on youth depression symptoms. Additionally, we hypothesized that depression is intergenerational in that depression in the mother's own mother plays a role in the mother's depression and in her stressful life context. We also explored whether there is a significant association between depression in the grandmother and in the youth.

2. We hypothesized that key mechanisms of the transmission of effects of maternal depression are the mother's quality of ongoing interpersonal relationships, which contribute to a stressful family context. These interpersonal relationships include the marital relationship with the husband/intimate partner, the psychiatric status of the child's father, and the mother's relationships with her own family and close friends. We also hypothesized that a link exists between maternal depression and maternal chronic interpersonal stress, with the latter predicting parenting quality, child social competence, and youth interpersonal stress. Additionally, we predicted that the relatively negative quality of a parent-child relationship will play an important role in youth depression.

3. The interpersonal transmission model proposes that the proximal predictors of youth depression are the child's own interpersonal relationships (social competence and stressful episodic life events to which the youth has contributed; events that are dependent at least in part on the youth's behavior and characteristics are commonly social in content). We hypothesized that it is maternal stressful interpersonal relationships and parent-child relationship quality that predict child interpersonal difficulties-both interpersonal competence generally and interpersonal stressful life events specifically.

4. Overall, therefore, maternal depression effects on youth depression are at least partially if not largely mediated by maternal interpersonal stress and parenting, and the effects of maternal interpersonal stress and parenting on youth depression in turn are at least partially mediated by their effects on the children's own interpersonal difficulties, which are the proximal predictors of depression.

Thus, the purpose of the present study was to test an intergenerational transmission model of depression, linking the depression of the mother and grandmother to stressful social circumstances that predict the youth's stressful social circumstances, which contribute to current depression. We also hypothesized, in addition to the specific paths that are predicted, that maternal depression's effects on youth depression are largely mediated by the stress and interpersonal factors.

It should be emphasized that the proposed model was not intended to account for all possible pathways to depression. Not only are the causes of depression varied given the assumption that there are multiple forms of depression, but the present study was not designed to test potentially genetic or other biological processes in intergenerational transmission, some of which may well operate through psychosocial pathways.

\section{Method}

\section{Participants}

The sample consisted of 816 adolescents (15 years of age) and their mothers. They were selected from a birth cohort originally consisting of 7,775 mothers and their children born between 1981 and 1984 at the Mater Misericordiae Mother's Hospital in Brisbane, Queensland, Australia (Keeping et al., 1989). The Mater-University of Queensland Study of Pregnancy (MUSP) was originally devised to investigate the children's physical, cognitive, and psychological health as a function of pregnancy and obstetric conditions, birth weight, and psychosocial conditions and to predict health, development, and behavior at age 5. The current article reports on data collected from a follow-up conducted when the target children were 15 years of age. 
Sample selection. At each of the four MUSP contacts (pregnancy, after delivery, and when the child was 6 months of age and 5 years of age), the mothers had completed a depression scale, the Delusions-Symptoms-States Inventory (DSSI; Bedford \& Foulds, 1978). The DSSI had been chosen as the measure of maternal mental health for the Mater Hospital birth cohort study because it was a valid screening instrument for mental health (e.g., Bedford \& Foulds, 1977) and did not include symptoms that might be confused with the effects of pregnancy or childbirth. The DSSI scores were used for screening for participation in the current project, and actual diagnostic information was collected in the present study as described below.

The present study was intended as an intensive follow-up of a large sample of depressed and nondepressed women and their children. Therefore, women's DSSI scores at the initial four testings were reviewed, and families were targeted for inclusion when their child became 15 years of age, on the basis of patterns of elevation of scores and their continuing residence in the Brisbane area (as determined by participation in the questionnaire follow-up of the original MUSP investigators when the child was 13 years of age). The goal was to oversample women with putative depressive disorders and to represent a range of depressive experiences varying in severity and frequency of elevated scores, along with a sample of comparison women who had no or few depressive symptoms. In order to get a continuous distribution of depressive experiences, we used a specific algorithm to select potential participants who varied in chronicity or severity of symptoms over the four testings (e.g., those who scored "severe depression" more than two times; those who scored only "moderately depressed" two or more times; those who scored "never severe"). Of the final group selected, $32 \%$ reported no or few depressive symptoms over the four testings, and $11 \%$ reported severe depression two or more times; all the others had scores in between these extremes, reflecting less severe or less chronic symptoms. As intended, these women represented a wide range of diagnosable depressive experiences by the time the children were 15 years of age, as noted below.

From the sample still available for follow-up at age 13 (5,277, or $68 \%$ of the original sample), 991 families were targeted for inclusion in the present study when the child was 15 years of age because of the depression scores of the mothers. Of the 991 families, 816 consented and were included (82\%); 68 families could not be located; 103 declined to participate in this wave; 3 included a child with a hearing or visual impairment that precluded participation; 1 child had died. Children in the sample were not significantly different from those in the original birth cohort in terms of gender, $\chi^{2}(1, N=7,775)=0.53, p=.48$; family income, $t(7773)=0.81$, $p=.42$; or mother's education, $t(7773)=1.70, p=.09$.

Characteristics of sample. There were 414 boys and 402 girls ( $M$ age $=$ 15 years 2 months, $S D=0.29$ ). Ninety-two percent of the overall sample were Caucasian, the median family income was in the lower range of middle class, the mothers' median education was Grade 10, and the mothers' mean age at the time of the youths' 15 -year follow-up was 41 years. Mothers' marital status included $76.8 \%$ who were currently married or cohabiting; overall, $64.8 \%$ were currently married to the biological father of the youth.

\section{Procedure}

Interviews were conducted in the homes of the families when the mother and the child — and when possible, the father-were available at the same time. Interviewers were blind to the mother's depression status or history, and a team of two interviewers conducted the parent and child interviews separately and privately. Between interviews, the participants also completed a battery of questionnaires, as noted below. The mother, child, and father gave written informed consent and were paid for their participation, which lasted approximately $3.5 \mathrm{hr}$.

A team of six interviewers was trained by one of the authors (Constance Hammen) to conduct the diagnostic evaluations and life stress interviews. All were advanced graduate students in clinical psychology at the University of Queensland and had prior clinical and research interview experience. They were trained to proficiency and were closely supervised via audiotape and periodic visits by the investigators. Systematic reviews of samples of interviews were conducted at 6-month intervals over the 3-year course of data collection to prevent drift. Specific procedures and interrater reliability information are provided below.

\section{Measures}

Maternal depression diagnosis. Maternal diagnostic information was based on the Structured Clinical Interview (SCID) for the fourth edition of the Diagnostic and Statistical Manual of Mental Disorders (DSM-IV; First, Spitzer, Gibbon, \& Williams, 1995). The interviewers, who were blind to the women's prior scores on the DSSI, ascertained the presence of lifetime and current diagnoses; the dating of onsets and episodes was as precise as possible. A reliability study based on 52 women in the study rated by independent judges yielded kappas of .87 for current diagnoses of MDE or dysthymic disorder and subsyndromal depression and .84 for past depressive diagnoses or symptoms.

The sample included 358 women (44\%) with current or past major MDE or dysthymic disorder up to the child's age of 15. One hundred sixty-four of the women had at least one period of dysthymic disorder, and 271 had at least one MDE (34\% of the total had two or more major depressive episodes). Four of the depressed women were diagnosed as having bipolar disorder ( 2 had a diagnosis of Bipolar I, and 2 had a diagnosis of Bipolar II) and were omitted from the analyses. Four hundred fifty-four women $(56 \%)$ had no history of diagnosable depression. Clinical ratings of maternal depression based on the lifetime SCID when the child was $0-5$ years of age corresponded significantly with DSSI categorizations of severity of depressive symptoms assessed contemporaneously during the same period, $\chi^{2}(1, N=812)=40.83, p<.01$, and recurrence (a proxy for chronicity) during the age $0-5$ interval, $\chi^{2}(1, N=812)=42.59, p<.01$. Maternal depression based on SCID interviews of past and current depression was scaled for the analyses $(0=$ never, $1=$ major depressive disorder or dysthymic disorder, 2 = both major depressive disorder and dysthymic disorder [either concurrent or at separate times]).

Youth diagnostic evaluation and self-reported depression. The presence of depressive disorders in the child was ascertained by means of the Schedule for Affective Disorders and Schizophrenia for School-Age Children-Revised (Epidemiologic version) for the DSM-IV (K-SADS-E; Orvaschel, 1995). The instrument is a semistructured interview covering current and lifetime disorders that is administered by trained clinical interviewers. It is administered separately to the parent and the child; diagnostic decisions were reviewed by the clinical rating team, with judgments based on all available information. The original K-SADS (e.g., Orvaschel, Puig-Antich, Chambers, Tabrizi, \& Johnson, 1982) reported excellent reliability and validity for use with clinical samples. Orvaschel (1995) reported that the $D S M-I V$ version had a kappa of .73 for current major depressive disorder and .72 for dysthymia, based on a clinical sample of 72 youngsters with a mean age of 11.6 years.

A reliability study in the current community sample was based on 75 interviews with the youths. Kappas were .82 for current depressive diagnoses (MDE or dysthymia) or subclinical depression and .73 for past depressive diagnoses or subclinical depression. Current depression diagnosis status was coded on a 3-point scale $(0=$ no significant depression, $1=$ subsyndromal depression [nearly meets criteria for MDE or dysthymic disorder], 2 = major depressive disorder or dysthymic disorder or both). Thirty-three youths met $D S M-I V$ criteria for current major depressive episode, dysthymic disorder, or both.

Self-reported current depressive symptoms were obtained with the Beck Depression Inventory (BDI; Beck, Ward, Mendelsohn, Mock, \& Erbaugh, 1961), a widely used and well-validated instrument for assessing the severity of depressive symptomatology. Nine percent of the sample scored 16 or above on the BDI. Additionally, mothers completed the Child Behavior Checklist (CBCL; Achenbach, 1991). These instruments have been well established as being reliable and valid measures of current symptomatology (Achenbach, 1991), and for the present study, the mother and youth broadband $T$ scores for internalizing (i.e., withdrawn, somatic complaints, anxiety/depression) symptoms were used. 
Youth's father and maternal grandmother diagnoses. Five hundred twenty-two fathers were directly interviewed with the SCID for lifetime diagnoses; of these, $454(87 \%)$ were the biological parents of the youth, $483(93 \%)$ lived in the home with both the mother and the youth, and 501 (96\%) reported that the youth lived with them at least some of the time. Reliability of father diagnoses on the basis of the SCID interviews was computed separately for past and current disorders in four categories (anxiety, depressive, substance use, and "other" disorders such as antisocial personality disorder). Kappas ranged between .72 for past anxiety disorders to .99 for current "other" (overall mean $\kappa=.85$ for current and .88 for past disorders). When the biological father of the child was not directly available for an interview, we used the Family History Research Diagnostic Criteria (FHRDC; Andreasen, Endicott, Spitzer, \& Winokur, 1977) to obtain reports from the mothers.

Mothers were interviewed about their parents' psychiatric status by means of the FHRDC method. FHRDC-based diagnostic reliabilities were obtained from 55 randomly selected informants $(\kappa=.80$ for diagnoses of the mother's mother [child's grandmother] and 1.0 for the biological fathers). For the present study, maternal grandmothers were coded as depression present or absent, and fathers were coded as present or absent any FHRDC diagnosis. Thirty-two women did not have any information on their biological mothers; of these, 13 were adopted and provided information on their adoptive mother. The remaining 19 were raised by others.

Maternal interpersonal stress. Chronic stress (ongoing conditions experienced over at least the past 6 months) was assessed via a semistructured interview covering typical functioning in key roles (e.g., Hammen et al., 1987). The instrument yields information that is a measure both of chronic stress in these areas and of current functioning in the past 6 months. The interview includes probes for three areas reported in the present study: quality of intimate (marital) relationships, close friendships, and relationships with extended family members (parents and siblings). Each domain is rated by the interviewer on a 5-point scale, with behaviorally specific anchor points indicating the severity of ongoing stressful conditions $(1=$ exceptionally good conditions, $5=$ extreme adversity). Interrater reliability based on independent judges' ratings ( $n=77-100$, depending on category) included intraclass correlations of .88 for current marital or intimate relationship, .82 close friend, and .77 extended family relationships. Convergent and construct validation have been obtained in various samples (e.g., Hammen et al., 1987; Rao et al., 1999).

Perceived parenting quality. Youths' reports of maternal parenting quality were derived from two questionnaires. One questionnaire contained two subscales measuring the child's perceptions of the mother's warmth and hostility, scored on 7-point scales $(1=$ always, $7=$ never $)$. The questionnaire was developed by the Iowa Youth and Families Project on the basis of their observational measures of the same constructs (e.g., Ge, Best, Conger, \& Simons, 1996), with high internal reliability and good correlations with observed parental warmth and hostility. In the present study, the 9-item Warmth scale had an internal consistency reliability of .91 , and the 15-item Hostility scale had an alpha of .92.

The second questionnaire included two subscales of the revised Children's Report of the Parental Behavior Inventory (CRPBI; Schludermann \& Schludermann, 1988): the Perceptions of Maternal Acceptance subscale and the Maternal Psychological Control subscale. The Acceptance scale consists of 10 items (e.g., "gives me a lot of care and attention"); the Psychological Control subscale also consists of 10 items ("is always telling me how I should behave"). The scales were scored on a 3-point scale ( $1=$ not like, 2 = somewhat like, 3 =lot like). Coefficient alphas in the present sample were .90 and .81 , respectively.

Youth social competence. Several interview- and questionnaireassessed variables measured aspects of youths' social functioning. The adolescents' ongoing experience in important roles was assessed with a semistructured interview modeled after that previously developed for adults (as described previously) and earlier versions of chronic strain/ functioning for children and adolescents (e.g., Hammen, 1991a). The adolescent version used in the present study consisted of six domains: social life, close friendship, romantic relationships (or dating interest), relations with family members, academic performance, and school behavior. Using standard general probes and follow-up queries when needed, interviewers probed each area with the youths. Each domain was scaled on a 5-point scale with behaviorally specific anchors $(1=$ represented superior functioning, $5=$ severe difficulties). For the present study, two scales concerning social functioning were summed: Close Friendship and Social Life (romantic relationships were not included because most of the 15 year-olds were not in such relationships). Reliabilities obtained from independent judges (intraclass correlations) were .76 and .63 , respectively.

The youths were administered the Self-Perception Profile for Adolescents (Harter, 1988), a 45-item self-report scale assessing domain-specific areas of competence. In order to parallel the interview-based measures of social functioning, two subscales, each consisting of five items, were included in the present study: Close Friendship (perceived ability to make close friends) and Social Acceptance (acceptance by peers, has friends, easy to like). Harter reported mean internal consistency reliabilities across four samples of .82 and .82 , respectively. Scores were summed across the five items of each scale to form totals, with higher scores representing more positive self-perceptions.

The Youth Self Report version of the Child Behavior Checklist (Achenbach, 1991) was administered, and three items from the Social Competence subscale that pertained to youths' perceptions of their friendships were summed for a social subscale.

Youth episodic interpersonal stress. Episodic stressful life events occurring in the past 12 months and judged by a rating team to be at least partly dependent on the youth's characteristics or behaviors were assessed via a semistructured episodic life stress interview procedure. On the basis of the Brown and Harris (1978) contextual threat approach, the interviewer probed the occurrence of specific events; dates of an occurrence were elicited if possible, and information was obtained about the nature of the event and the circumstances in which it occurred. Interviewer-prepared narratives of each event were presented to a rating team that was blind to the youth's family status and actual reactions to the event. The team rated each event on two scales: Severity (how much impact the event would have on a typical person under similar conditions, rated on a 5-point scale, with 5 indicating extremely severe), and Independence (the extent to which the occurrence of the event was independent or dependent on behaviors or characteristics of the individual, rated on a 5-point scale but dichotomized in the present study as being independent, or at least as being partly dependent, on the person).

With the current study's focus on episodic stressful events to which the youth has at least partly contributed as a result of his or her interpersonal characteristics, competence, and behaviors, a summary score for the total stress of all dependent events in the past year was used in the analyses. Interrater reliabilities based on separate ratings by Australian and U.S teams for 89 cases yielded intraclass correlations of .92 for the severity rating and .89 for the independence rating. Total dependent stress scores ranged from 0 to $15.5(S D=2.9)$, with a median of 2.5. Seventeen percent of the sample scored 5.0 or more, and 33 youths had at least one severe (rated 3 or higher) event.

\section{Results}

\section{Overview of Analyses}

Structural equation modeling (SEM) tests hypotheses at the construct level rather than at the level of measured variables. Therefore, measurement errors associated with any one particular instrument are minimized. Furthermore, constructs can be derived from several reporting sources and methods (e.g., mother, child, and interviewer reports, as well as scores from questionnaires and interview-based ratings). By extracting the shared variance among the measured variables to create a latent variable, measurement and reporter biases within one measure are minimized in the latent construct. Wherever possible, constructs in this study were derived from multiple sources and methods. Another advantage of using 


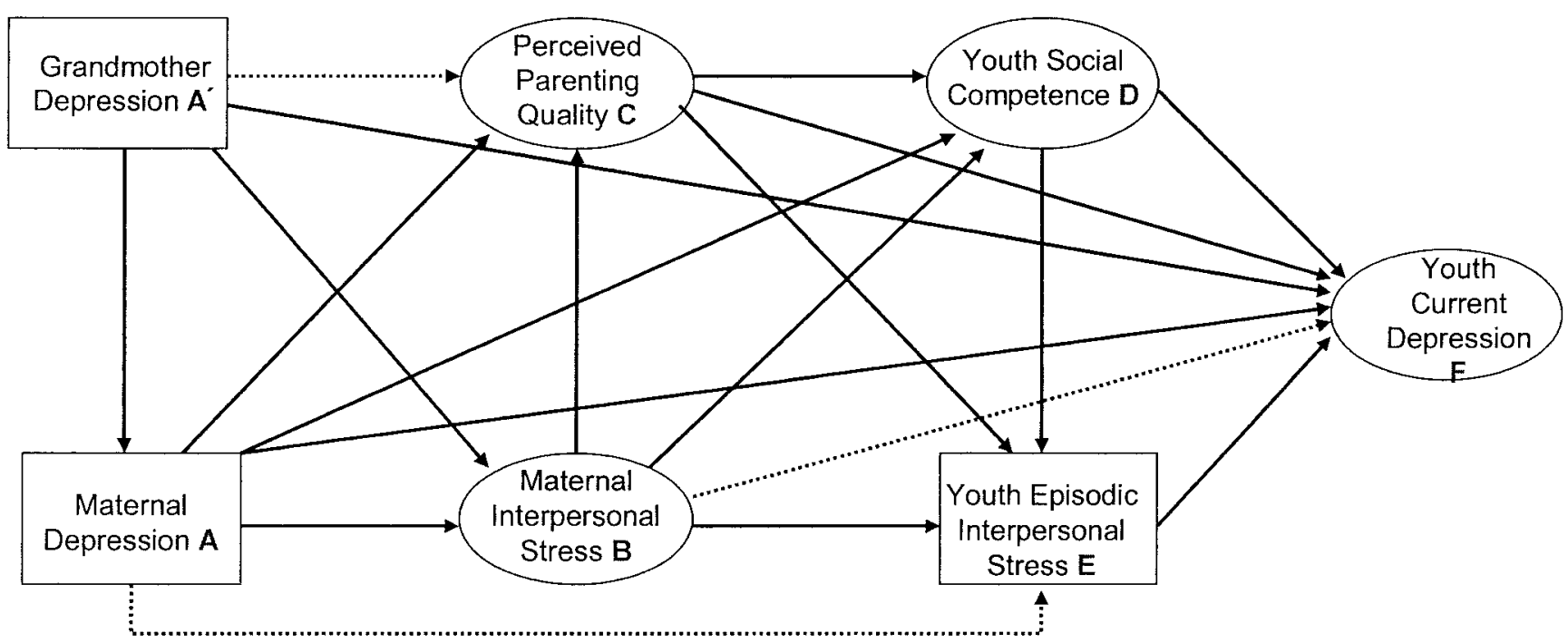

Figure 1. Hypothesized structural model on the intergenerational transmission of depression. Rectangles represent measured variables; ovals represent latent variables. Dotted lines indicate direct paths not specifically predicted in the theoretical model but that are included in this model to fully test mediation hypotheses. This figure is referred to as the "full model" in mediation analyses.

SEM is its ease of testing complex mediational relationships, because it allows for the testing not only of $\mathrm{A} \rightarrow \mathrm{B} \rightarrow \mathrm{C}$ but also of complicated mediations that have more than three variables.

Program and estimation method. The structural equation models were tested using the EQS program (Bentler, 2002). The robust maximum likelihood estimation method was used in the present analyses to adjust for univariate and multivariate nonnormality in the data (Byrne, 1994). For each model, three test statistics are reported: the Yuan-Bentler-scaled chi-square (Y-B $\left.\chi^{2}\right)$, the comparative fit index (CFI), and the root-mean-square error of approximation (RMSEA; Browne \& Cudeck, 1993; Hu, Bentler, \& Kano, 1992; Ullman, 1996; Yuan \& Bentler, 2000). Similar to other chi-square test statistics in SEM, the Y-B $\chi^{2}$ measures the discrepancy between the population covariance matrix estimated from the model and the sample covariance matrix. That is, the statistic indicates how closely the path coefficients of the model in our sample compare with what would be expected in the population. In addition, the Y-B $\chi^{2}$ adjusts for nonnormal data (similar to the chi-square used by Satorra $\&$ Bentler, 1988) and can be implemented when data are missing (Yuan \& Bentler, 2000).

Because trivial differences between the matrices are often significant when the sample size is large, a comparison-based fit index (CFI) and a residual-based fit index (RMSEA) were also evaluated. CFI values range from 0 to 1 , with values of .95 or greater accepted as indicating a good model fit (Hu \& Bentler, 1999). RMSEA values range from 0 to 1 , with values less than .06 accepted as indicating good model fit (Hu \& Bentler, 1999). In addition, during the model modification phase, the Lagrange Multiplier Test was used to identify potential paths that would improve model fit (Chou \& Bentler, 1990), and the Wald test statistic, which indexes the amount that a model's overall chi-square would increase if a particular path is dropped from the model, was used to identify potential extraneous paths that can be removed without degrading model fit (Kline, 1998).

Sample inclusion/exclusion. Of the 816 participating families, $67 \mathrm{had}$ one or more missing data points. Missing data were present in the following study variables: the maternal chronic stress interview $(n=2 ; 0.02 \%)$, the Warmth/Hostility scales $(n=8 ; 1 \%)$, the CRPBI $(n=8 ; 1 \%)$, dependent interpersonal episodic stress $(n=$ $3 ; 0.04 \%)$, the youth social competence interview $(n=1 ; 0.01 \%)$, the CBCL friendship items $(n=11 ; 1 \%)$, the Harter self-concept scale $(n=11 ; 1 \%)$, maternal report of the CBCL-Internalizing scale $(n=29 ; 4 \%)$, and youth-reported BDI $(n=11 ; 1 \%)$.

In the present study, the majority of the missing data can be construed as missing at random, mostly because of time limitations or equipment failure (laptop self-entry of questionnaire data). The maximum likelihood expectation maximization (MLEM) method was used to impute values for variables with missing data (Bentler, 2002; Jamshidian \& Bentler, 1999) except for several cases of maternal grandmother depression, which were omitted from analyses. $^{1}$ Overall, 67 families had one or more missing values; 48 families were retained with imputation of their randomly missing values, and 19 were omitted from the analyses.

Structural model and model indicators. The structural model tested is presented in Figure 1. This model includes four latent variables and three manifest variables. The following are the labels and contents of the model indicators:

\footnotetext{
${ }^{1}$ Several cases of FHRDC-based maternal grandmother depression $(N=$ $19 ; 2 \%$ ) could not be considered missing at random, as some of the women had no information to provide about biological or adoptive mothers (raised by others) and were dropped from the present analyses. The 19 excluded women did not differ on history of depression, nor did their children differ on history of diagnosis of depression compared with the remainder of the sample. The 19 youths did not differ on their BDI scores, but the excluded mothers viewed their children as having more internalizing symptoms on the CBCL $(M \mathrm{~s}=6.4$ vs. 10.2 for included and excluded youths, respectively), $t(785)=2.49, p=$ .01 . They and their children did not differ on any other predictor variables with two exceptions: Excluded mothers had significantly worse interviewer-rated relationships with friends and extended family members, and their children had better interviewer-rated social relationships.
} 
Grandmother depression is a manifest variable derived from FHRDC interviews of the mother $(0=$ absent, $1=$ present $)$. Maternal depression is a manifest variable derived from the SCID interview ( $0=$ no history of depression, $1=$ history of $M D D$ or dysthymic disorder, $2=M D D$ and dysthymic disorder).

Maternal chronic interpersonal stress is a latent factor indicating the presence of any diagnosable disorder in the child's biological or current step-father, plus three interview-based chronic stress ratings: current marital relationship, relationships with extended family, and quality/presence of close friendship. All factor indicators were coded so that higher values indicated more interpersonal stress.

Perceived parenting quality is a latent factor with four indicators: youth reports of CRPBI Maternal Acceptance, CRPBI Psychological Control, and the Warmth and Hostility scales. All four indicators have been coded so that higher values indicate better perceived parenting quality. On the basis of the results of the Lagrange Multiplier Test, one set of correlated error residuals was added to this factor. The residuals of the Warmth and Acceptance scales were freed to correlate. Their correlation suggests that the Warmth and Acceptance scales were correlated over and above the common variance in perceived parenting quality.

Youth social competence is a latent factor with three indicators: youth-report CBCL items on friendships; combined Harter scales of Social Acceptance and Close Friendship, and the sum of interviewer-rated social life and presence/quality of best friend. All three indicators were coded so that higher values indicated greater social competence.

Youth episodic interpersonal stress is a manifest variable derived from the episodic stress interview procedure. The objective severity ratings of stressors that were deemed to be interpersonal and dependent (caused at least in part by the person) were summed for the present analyses. Higher values indicated greater stress.

Youth current depression is a latent variable with three indicators: SCID-based current diagnoses of depressive disorders (as indicated in Method section), self-report BDI scores, and motherreported CBCL Internalizing composite score. Higher values on this factor indicated more severe depression and distress.

The observed intercorrelations among the measured variables in the study are presented in Table 1 . The model correlation matrix for latent and measured variables is presented in Table 2.

Fit of structural model. Results from the SEM analyses indicated that the specified model (see Figure 1) with all the significant predicted direct and indirect paths provided an acceptable fit to the data, Y-B $\chi^{2}(102, N=797)=201.11, p<.05$; CFI $=.96$; and RMSEA $=.04$. To aid parsimony, we analyzed a reduced model (see Figure 2), dropping most of the nonsignificant paths in the overall model in the order suggested by the Wald test. ${ }^{2}$ This reduced model provided a good fit, Y-B $\chi^{2}(106, N=797)=$ 204.48, $p<.05$; CFI $=.96$; and RMSEA $=.04$. The chi-square difference test indicated that the reduced model did not compromise the fit of the original specified model: Y-B $\chi_{\text {diff }}^{2}(4, N=$ $797)=3.37(n s)$. The path coefficients of the original and reduced models were highly correlated at $.97(p<.01)$. This is another indication that the paths in the reduced model very closely mirrored those of the original model and that the relationships found in the original model were retained despite the model modifications.
The results of the parsimonious model are presented in Figure 2. The following are the significant relationships observed in the model:

1. Grandmother depression predicted maternal depression and increased maternal chronic interpersonal stress.

2. Maternal depression predicted increased maternal chronic interpersonal stress. Maternal depression predicted lower youth social competence in the parsimonious model, as noted earlier (see Footnote 2).

3. Higher maternal interpersonal stress predicted poorer perceived parenting quality, higher youth interpersonal episodic stress, and youth depression.

4. Poorer perceived parenting quality predicted increased youth interpersonal episodic stress and lower youth social competence. Poorer perceived parenting quality predicted increased youth depression.

5. Higher youth social competence predicted less youth depression but an increase in youth episodic interpersonal stress. Although counter to expectations, the results imply that greater sociability may be associated with more chances for social stressors.

6. Higher youth episodic interpersonal stress predicted greater youth depression.

To summarize, in both of the models tested, the following hypothesized relationships were observed: Youth depression was predicted by the proximal youth interpersonal factors (youth interpersonal stress and youth social competence). Youth depression was also predicted by the more distal family environment factors of maternal chronic interpersonal stress and perceived parenting quality. Grandmother depression and maternal depression did not predict youth depression in the full model. This does not necessarily indicate that there is no relationship between grandmother/ maternal depression and youth depression; rather, these findings suggest that the relationship between grandmother/maternal depression and youth depression may be fully mediated as proposed by the model, and tested below.

\section{Overview of Mediation Analyses}

An important component of the model is the question of whether the effects of maternal (and grandmother) depression on youth depression are largely accounted for by interpersonal stress mechanisms. To ascertain the specific mediators that facilitate the intergenerational transmission of depression, we followed Baron and Kenny's (1986) guidelines to test the mediation hypotheses.

\footnotetext{
${ }^{2}$ For additional information regarding the model reduction, including tables with test statistics, please contact Josephine Shih at jhshih@ ucla.edu. The dropped paths included grandmother depression to perceived parenting quality, maternal depression to youth episodic interpersonal stress, maternal interpersonal stress to youth social competence, and maternal depression to perceived parenting quality. Of note, the paths from grandmother depression to youth depression and from maternal depression to youth depression were not dropped from the analyses so that the intergenerational transmission of depression hypothesis could be properly evaluated. In addition, the nonsignificant path from maternal depression to youth social competence $(\beta=-.09, z=-1.42, p<.15)$ was not dropped. This path appears to be important to the model because the Wald test did not recommend its deletion, and dropping it significantly degraded the model fit. In fact, this path is statistically significant in the reduced model $(\beta=-.13, z=-2.60, p<.05)$.
} 


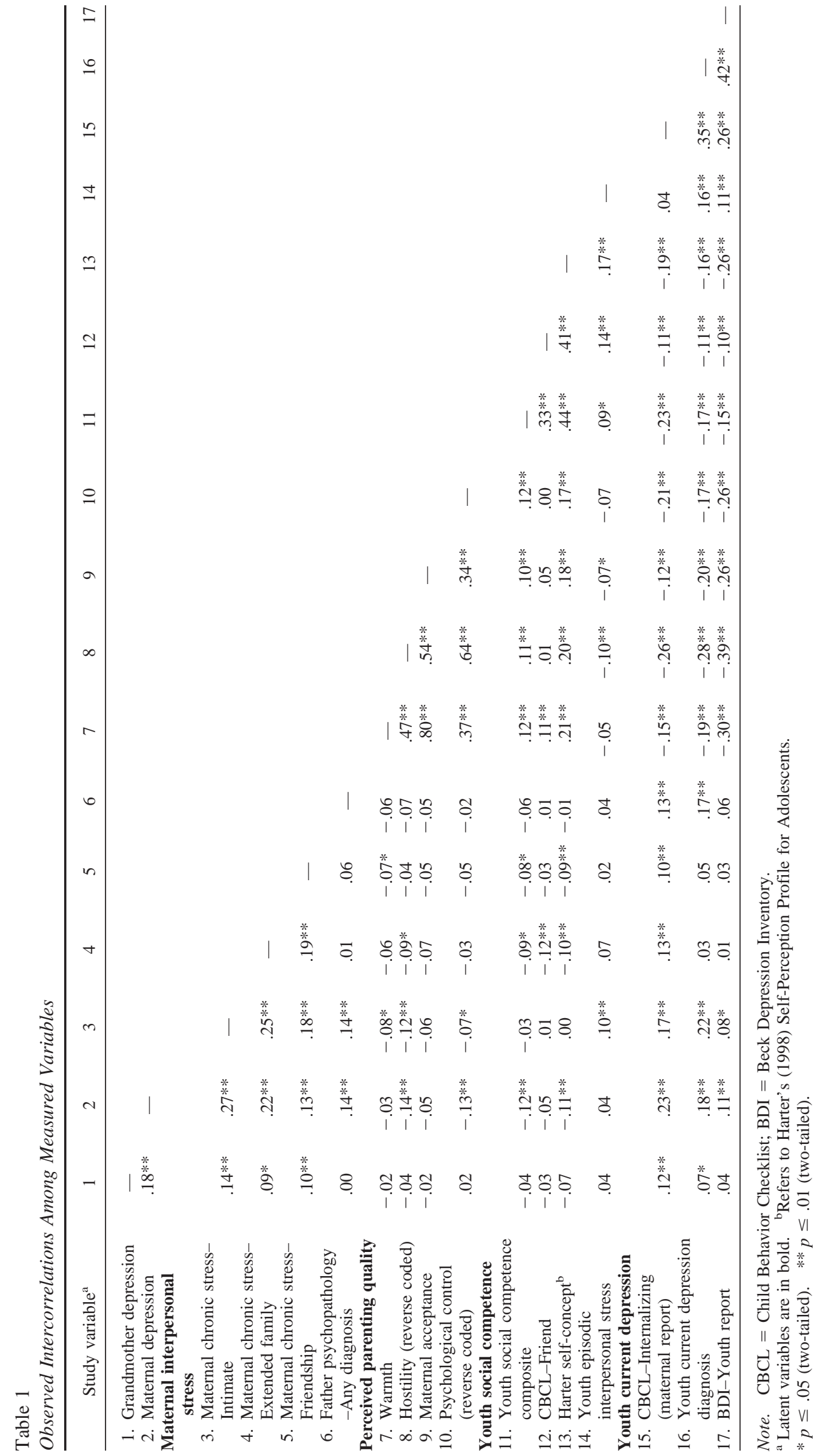


Table 2

Model Correlation Matrix for Latent and Measured Variables

\begin{tabular}{|c|c|c|c|c|c|c|c|}
\hline Measured or latent variable & 1 & 2 & 3 & 4 & 5 & 6 & 7 \\
\hline 1. Grandmother depression & - & & & & & & \\
\hline 2. Maternal depression & .18 & - & & & & & \\
\hline 3. Maternal interpersonal stress & .22 & .49 & - & & & & \\
\hline 4. Perceived parenting quality & -.05 & -.12 & -.24 & - & & & \\
\hline 5. Youth social competence & -.03 & -.15 & -.11 & .23 & - & & \\
\hline 6. Youth episodic interpersonal stress & .03 & .05 & .16 & -.11 & .21 & - & \\
\hline 7. Youth current depression & -.11 & .26 & .38 & -.56 & -.43 & .19 & - \\
\hline
\end{tabular}

The mediations tested in the present study are complicated by the inclusion of multiple mediators. Thus, one of several mediation paths the model tests is akin to $\mathrm{A} \rightarrow \mathrm{B} \rightarrow \mathrm{C} \rightarrow \mathrm{D} \rightarrow \mathrm{E} \rightarrow \mathrm{F}$ (e.g., maternal depression $\rightarrow$ maternal stress $\rightarrow$ parent quality $\rightarrow$ social competence $\rightarrow$ youth episodic interpersonal stress $\rightarrow$ youth depression). In a stringent test of mediation, the present analyses not only required that the $\mathrm{AF}$ path be significant but also that $\mathrm{A}$ directly predict $\mathrm{C}, \mathrm{D}$, and $\mathrm{E}$ as well. This is an extension of Holmbeck's (1997) differentiation of indirect and mediated effects.
Maternal and youth depression. To test the mediation hypothesis that maternal depression's effect on youth depression $(F)$ is largely mediated through maternal stress (B), parenting quality (C), youth social competence (D), and youth interpersonal stress (E), AC, AD, AE, and AF paths need to be established as significant and in the predicted direction. The full model indicated that maternal depression predicts increased maternal interpersonal stress as hypothesized (AB path, $\beta=.46, z=7.92, p<.01$ ). Examination of pure models as defined earlier indicated that maternal depression directly predicted perceived parenting quality

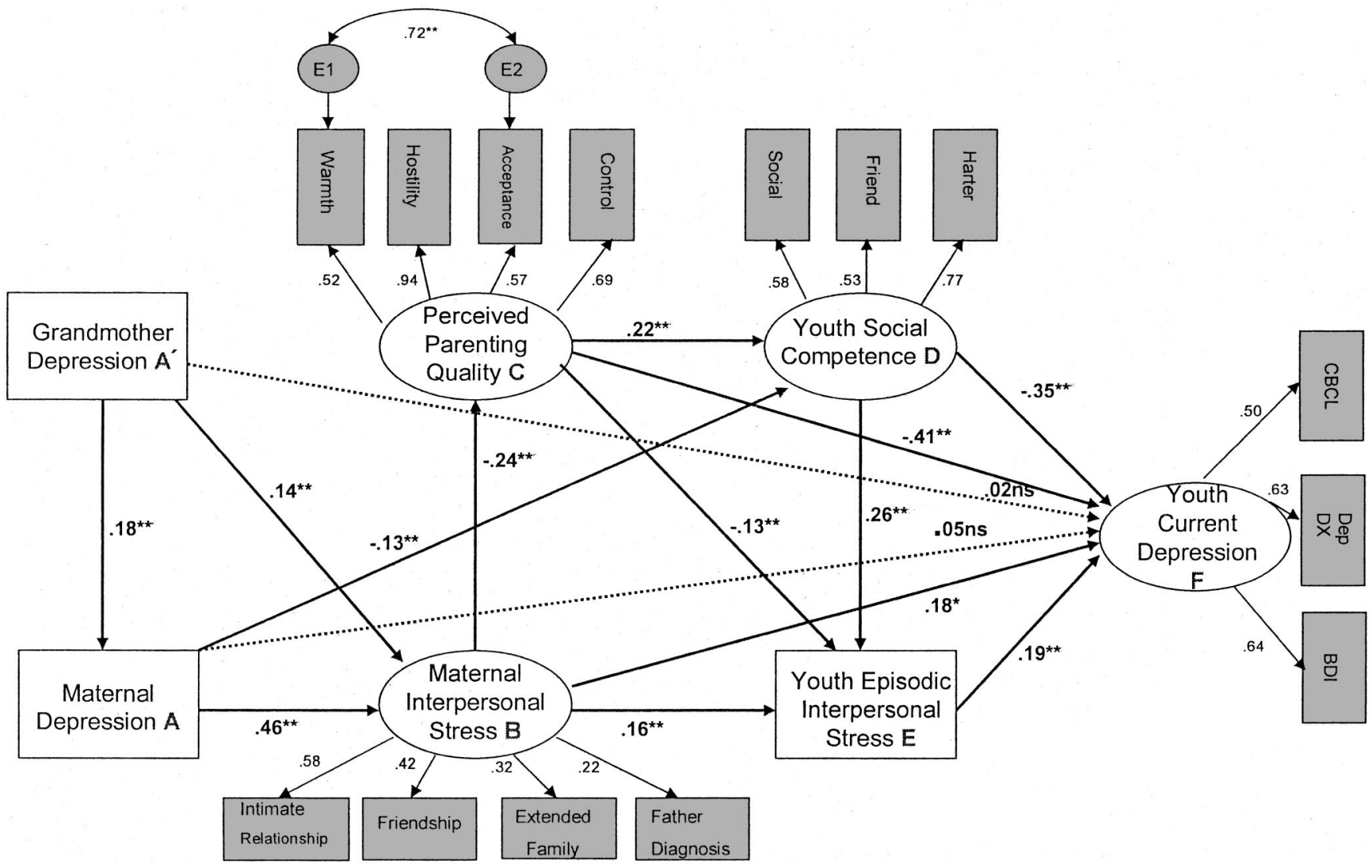

Figure 2. Reduced structural model on the intergenerational transmission of depression $(N=797)$, with standardized parameter estimates. Nonsignificant paths from the full model were deleted except for the two direct paths (the dotted lines in this figure) that were necessary to fully test the intergenerational transmission hypothesis. $\mathrm{CBCL}=$ Child Behavior Checklist; Dep DX = diagnoses of depressive disorders based on the Structured Clinical Interview; BDI $=$ Beck Depression Inventory. All factor loadings were significant at $p<.01$. For all other path parameters: $* p<.05 . * * p .01$. 
(AC path, $\beta=-.15, z=-3.458, p<.01$; Y-B $\chi^{2}[4, N=797]$ $=9.01, n s ; \mathrm{CFI}=.996$; and RMSEA $=.04)$, youth social competence (AD path, $\beta=-.16, z=-3.05, p<.01$; Y-B $\chi^{2}[2$, $N=797]=2.17, n s ; \mathrm{CFI}=.999$; and RMSEA $=.01)$, and youth depression (AF path, $\beta=-.26, z=4.11, p<.01$; Y-B $\chi^{2}[2, N=$ 797] $=9.21, p<.05$; CFI $=.952$; and RMSEA $=.067)$. However, maternal depression did not predict youth episodic interpersonal stress (AE path, $r=.04, n s$ ). Next, an examination of parameter indirect effects indicated that the maternal depression to youth depression path was significantly reduced with the addition of the mediators $(\beta=.19, z=3.59 p<.01)$.

The results suggest that maternal depression does not predict youth depression through the effect of maternal depression on youth episodic interpersonal stress. Instead, the results indicate that, as hypothesized, the relationship between maternal depression and youth depression is largely mediated through maternal depression's effect on maternal stress as well as through parenting quality and youth social competence. Examination of the significance and direction of the predicted paths indicates that the following are mediated pathways for the intergenerational transmission of depression from mothers to their children:

1. Maternal depression $\rightarrow$ maternal stress $\rightarrow$ youth depression

2. Maternal depression $\rightarrow$ maternal stress $\rightarrow$ parenting quality $\rightarrow$ youth depression

3. Maternal depression $\rightarrow$ maternal stress $\rightarrow$ parenting quality $\rightarrow$ social competence $\rightarrow$ youth depression

4. Maternal depression $\rightarrow$ youth social competence $\rightarrow$ youth depression

It should be noted that maternal depression significantly predicted youth depression in the pure model $(\beta=.26, z=4.11, p<$ $.01)$, but this relationship was not significant $(\beta=.05, z=.92)$ when mediators were introduced in the full model presented in Figure 1. This pattern of results indicates that the transmission of depression from mothers to their children is fully mediated by maternal stress, parent quality, and social competence.

Grandmother and youth depression. The same pattern of results was obtained for grandmother and youth depression. That is, the association between grandmother and youth depression was fully mediated by the effect of grandmother depression on maternal depression and maternal interpersonal stress. Grandmother depression $\left(\mathrm{A}^{\prime}\right)$ predicted an increase in youth depression $(\mathrm{F})(\beta=$ $.11, z=2.19, p<.05 ; \mathrm{Y}-\mathrm{B} \chi^{2}[2, N=797]=3.58, n s ; \mathrm{CFI}=$ .989 ; and RMSEA $=.03$ ) in the pure model, but its effect was reduced to nonsignificance $(\beta=.02, z=.42$ ) when the mediators were included in the full model. To test the mediation hypothesis that the effect of grandmother depression $\left(\mathrm{A}^{\prime}\right)$ on youth depression (F) is largely mediated through the effects of grandmother depression on maternal depression (A), maternal interpersonal stress (B), parenting quality (C), youth social competence (D), and youth interpersonal stress $(\mathrm{E})$, paths $\mathrm{A}^{\prime} \mathrm{B}, \mathrm{A}^{\prime} \mathrm{C}, \mathrm{A}^{\prime} \mathrm{D}, \mathrm{A}^{\prime} \mathrm{E}$, and $\mathrm{A}^{\prime} \mathrm{F}$ need to be established as significant and in the predicted direction. The full model indicates that grandmother depression predicted increased maternal depression (A'A path, $\beta=.18, z=4.72, p<$ $.01)$. Examination of the pure model indicates that grandmother depression predicted maternal stress ( $\mathrm{A}^{\prime} \mathrm{B}$ path, $\beta=.23, z=3.83$, $p<.01$; Y-B $\chi^{2}[5, N=797]=10.50, n s ; \mathrm{CFI}=.953$; and RMSEA $=.04)$. However, grandmother depression did not predict parenting quality ( $\mathrm{A}^{\prime} \mathrm{C}$ path, $\beta=-.04, z=-.97, n s$; Y-B $\chi^{2}[4$, $N=797]=9.38, n s ; \mathrm{CFI}=.996$; and RMSEA $=.04)$, youth social competence ( $\mathrm{A}^{\prime} \mathrm{D}$ path, $\beta=-.08, z=-1.73, n s$; Y-B $\chi^{2}[2$, $N=797]=0.61, n s ; \mathrm{CFI}=1.00$; and RMSEA $=.00)$, or youth episodic interpersonal stress ( $\mathrm{A}^{\prime} \mathrm{E}$ path, $\beta=.04, z=1.16, n s$ ). Examination of the significance and direction of the predicted paths (see Figure 2) indicates that the following are mediated pathways for the intergenerational transmission of depression from grandmothers to their grandchildren:

1. Grandmother depression $\rightarrow$ maternal depression $\rightarrow$ maternal interpersonal stress $\rightarrow$ youth depression

2. Grandmother depression $\rightarrow$ maternal interpersonal stress $\rightarrow$ youth depression

\section{Discussion}

Current depressive symptoms and disorders in 15-year-old youths were predicted in a complex model of interpersonal and family stressors in the context of two generations of maternal depression. The four hypothesized components of an intergenerational, interpersonal stress model of depression were supported. First, the effect is intergenerational: The grandmother's depression affected the mother's depression and her own stressful life context, and maternal and grandmother depression affected youth depression as mediated by interpersonal stress processes. Second, key mechanisms of the process are interpersonal and include maternal interpersonal stress and functioning, which in turn affects parenting and children's social functioning. Third, youth depression is proximately predicted by youth interpersonal stress, which itself was influenced by maternal interpersonal processes (stress and parenting), affecting youth social competence. Finally, the link between maternal (and grandmother) and child depression was entirely mediated by interpersonal and contextual factors.

The model that was proposed and supported by data from nearly 800 families indicated that maternal (and grandmother) depression are important risk factors for youth depression but that their effects operate through mechanisms of chronic maternal interpersonal stress (including disorder in fathers, as well as marital and family discord) and parenting quality that is perceived by the youngsters as relatively negative. Exposure to maternal interpersonal and parenting difficulties predicts depression and affects youths' social competence and experience of negative interpersonal life events, stressors that are proximal predictors of youth depression. Both the overall model with specifically predicted paths and the tests of mediation were statistically significant.

The findings are consistent with our intergenerational interpersonal stress model of depression transmission, in which women's depressive experiences are typically embedded in highly stressful family contexts (Hammen, 2002; Hammen \& Brennan, 2002). Heretofore it has been difficult to disentangle the correlated effects of depression and stressors in high-risk studies because of limited sample sizes and lack of availability of an array of variables that capture multiple areas of family and youth functioning. Use of SEM permits complex hypotheses to be tested. The present study provided results suggesting that maternal depression is an indicator 
of interpersonal discord and stress that affects the child's own interpersonal functioning and exposure to stressful events, which in turn trigger depression. Elsewhere we demonstrated that adverse family and environmental conditions are relatively stable, even when women are not currently depressed (e.g., Hammen \& Brennan, 2002). It may be that although symptoms of depression undoubtedly impair women's functioning in marital and parental roles, it is the relatively stable impairments and dysfunctions in their social lives that portend risk to children.

The findings of the importance of family and environmental context factors are generally consistent with a few offspring studies that have included measures of family risk factors (e.g., Billings \& Moos, 1985; Fergusson, Horwood, \& Lynskey, 1995; Goodman et al., 1993; Seifer et al., 1996). Similarly, Diamond and Doane (1994) linked mothers' attachment difficulties with their own mothers to the negative affective styles they displayed in interactions with their disturbed children. In contrast, while confirming the existence of highly adverse family environments in the families of children of depressed parents, Fendrich, Warner, and Weissman (1990; see also Nomura, Wickramaratne, Warner, Mufson, \& Weissman, 2002) found that family risk factors were predictive of depression in the offspring of nondepressed parents, whereas parental depression was more important in the high-risk families. It might be noted that Warner et al. (1995) found that in addition to parental depression, coparent alcohol abuse predicted youth depression, and chaotic family environment predicted offspring dysthymic disorder-findings potentially consistent with the current results.

At first glance the results of the present study might seem to suggest that genetic factors are relatively less important factors of intergenerational transmission of depression than are psychosocial mechanisms. However, it is important to acknowledge that some of the processes that are significant in the current model, such as interpersonal discord and stress occurrence, may themselves have a genetic component (e.g., reviewed in Goodman \& Gotlib, 1999; Wallace, Schneider, \& McGuffin, 2002). It is possible and indeed likely that heritable dispositional factors may contribute to the creation of negative social environments that trigger depression in both mothers and their children. Thus, unmeasured genetic factors may play an important role in understanding the mediational associations between parent and child depression.

In addition to the conceptual implications, the current findings have implications for treatment of youths at risk because of parental depression. They suggest that although parental depressive episodes may be transitory, enduring family difficulties and youth social competence may be important targets of intervention.

Several limitations of the current study are noted. Only 15-yearolds from an Australian community sample were included. It is possible that different models would apply to samples that are significantly younger or older. Although Australian culture is similar in many ways to that of the United States, there may be limitations in the generalizability to U.S. samples. Similarly, the study included a nonclinical maternal sample, and it included both diagnoses and subsyndromal measures of youth depression. The model might not apply to a more severely ill sample of depressed mothers or to youth who meet full criteria for current major depressive disorder. However, subclinical symptoms of depression have clearly been shown to have strong associations with impaired functioning and future depressive episodes (e.g., Angst, Sellaro, \& Merikangas, 2000; Gotlib, Lewinsohn, \& Seeley, 1995; Hays,
Wells, Sherbourne, Rogers, \& Spritzer, 1995; Horwath, Johnson, Klerman, \& Weissman, 1992). Therefore, we expect that youth depression as modeled in the current study will have important consequences for future functioning and psychopathology.

The current data were cross-sectional; however, maternal and grandmother depression variables preceded current symptoms in the youths, and the youth and family functioning variables reflected relatively stable factors likely existing for many years (see, e.g., Hammen \& Brennan, 2002, regarding the stability of maternal functioning factors). A longitudinal study that is currently underway on the present sample may provide future opportunity to evaluate the intergenerational interpersonal stress model. One latent variable comprised self-reports (perceptions of parents' behavior) that could reflect bias due to current depression, although interviews of mothers confirmed the relatively poorer quality of relations between depressed mothers and their children. In general, SEM and our use of multiple informants and measures guard against bias by extracting the shared variance among the measured variables to create a latent variable.

There may be limitations in the model presented, inasmuch as some variables that might be important were not measured or included (e.g., genetic, neurobiological processes in stress reactivity, as well as potentially important psychosocial mechanisms such as transmission of dysfunctional cognitions about the self and world). Also, although the present model presents a general view of how maternal depression eventuates in youth depression, further research on the precise mechanisms is needed-for example, one question might concern how maternal parenting quality may affect youth social competence and stress generation. Further, the current model is tested on a sample of families varying in maternal depression. Elsewhere we have shown that the youths in the study may develop depressive disorders whether or not their mothers are depressed (e.g., Hammen \& Brennan, 2001) and have proposed that there are likely different pathways to depressive outcomes in adolescence. The present model was a model of adolescent depression outcomes depending on the presence or absence of maternal depression, but it is possible that samples comprised entirely of those without maternal depression might yield different results.

Overall, the present study tested a complex model of intergenerational transmission of depression via family environment and youth stress processes and demonstrated that such factors mediated the link between mother and adolescent offspring depression. The study addresses a significant gap in the research on high-risk offspring (e.g., Goodman \& Gotlib, 1999), but many unresolved questions about mechanisms and future outcomes remain to be pursued.

\section{References}

Achenbach, T. M. (1991). The derivation of taxonomic constructs: A necessary stage in the development of developmental psychopathology. In D. Cicchetti \& S. Toth (Eds.), Rochester Symposium on Developmental Psychopathology: Models and integrations (Vol. 3, pp. 43-74). Hillsdale, NJ: Erlbaum.

Adrian, C., \& Hammen, C. (1993). Stress exposure and stress generation in children of depressed mothers. Journal of Consulting and Clinical Psychology, 61, 354-359.

Andreasen, N. C., Endicott, J., Spitzer, R. L., \& Winokur, G. (1977). The family history method using diagnostic criteria. Archives of General Psychiatry, 34, 1229-1235. 
Angst, J., Sellaro, R., \& Merikangas, K. (2000). Depressive spectrum diagnoses. Comprehensive Psychiatry, 41(Suppl. 1), 39-47.

Baron, R. M., \& Kenny, D. A. (1986). The moderator-mediator variable distinction in social psychological research: Conceptual, strategic, and statistical considerations. Journal of Personality and Social Psychology, $51,1173-1182$

Beardslee, W. R., Versage, E. M., \& Gladstone, T. R. (1998). Children of affectively ill parents: A review of the past 10 years. Journal of the American Academy of Child and Adolescent Psychiatry, 37, 1134-1141.

Beck, A. T., Ward, C. H., Mendelsohn, M., Mock, J., \& Erbaugh, J. (1961). An inventory for measuring depression. Archives of General Psychiatry, 4, 561-571.

Bedford, A., \& Foulds, G. (1977). Validation of the Delusions-SymptomsStates Inventory. British Journal of Medical Psychology, 50, 163-171.

Bedford, A., \& Foulds, G. (1978). Delusions-Symptoms-States Inventory of Anxiety and Depression. Windsor, England: NFER.

Bentler, P. M. (2002). EQS 6 structural equation program [Computer software and manual]. Encino, CA: Multivariate Software.

Billings, A. G., \& Moos, R. H. (1985). Psychosocial processes of remission in unipolar depression: Comparing depressed patients with matched community controls. Journal of Consulting and Clinical Psychology, 53, 314-325.

Brown, G. W., \& Harris, T. O. (1978). Social origins of depression. London: Free Press.

Brown, G. W., \& Harris, T. O. (1989). Depression. In G. W. Harris \& T. O. Harris (Eds.), Life events and illness (pp. 49-93). New York: Guilford Press.

Browne, M. W., \& Cudeck, R. (1993). Alternative ways of assessing model fit. In K. A. Bollen \& J. S. Long (Eds.), Testing structural models (pp. 136-162). Newbury Park, CA: Sage.

Byrne, B. M. (1994). Structural equation modeling with EQS and EQS/ Windows: Basic concepts, applications, and programming. Thousand Oaks, CA: Sage.

Chou, C. P., \& Bentler, P. M. (1990). Model modification in covariance structure modeling: A comparison among likelihood ratio, Lagrange Multiplier, and Wald tests. Multivariate Behavioral Research, 25, 115136.

Cummings, E. M., \& Davies, P. T. (1994). Maternal depression and child development. Journal of Child Psychology \& Psychiatry \& Allied Disciplines, 35, 73-112.

Diamond, D., \& Doane, J. (1994). Disturbed attachment and negative affective style: An intergenerational spiral. British Journal of Psychiatry, 164, 770-781.

Downey, G., \& Coyne, J. C. (1990). Children of depressed parents: An integrative review. Psychological Bulletin, 108, 50-76.

Fendrich, M., Warner, V., \& Weissman, M. M. (1990). Family risk factors, parental depression, and psychopathology in offspring. Developmental Psychology, 26, 40-50.

Fergusson, D., Horwood, L. J., \& Lynskey, M. (1995). Maternal depressive symptoms and depressive symptoms in adolescents. Journal of Child Psychology and Psychiatry, 36, 1161-1178.

First, M. B., Spitzer, R. L., Gibbon, M., \& Williams, J. B. W. (1995). Structured Clinical Interview for DSM-IV Axis I disorders. Washington, DC: American Psychiatric Press

Ge, X., Best, K. M., Conger, R. D., \& Simons, R. L. (1996). Parenting behaviors and the occurrence and co-occurrence of adolescent depressive symptoms and conduct problems. Developmental Psychology, 32, 717-731.

Gelfand, D. M., \& Teti, D. M. (1990). The effects of maternal depression on children. Clinical Psychology Review, 10, 329-353.

Goodman, S. H., Brogan, D., Lynch, M. E., \& Fielding, B. (1993). Social and emotional competence in children of depressed mothers. Child Development, 64, 516-531.

Goodman, S., \& Gotlib, I. (1999). Risk for psychopathology in the children of depressed mothers: A developmental model for understanding mechanisms of transmission. Psychological Review, 106, 458-490.

Gotlib, I. H., Lewinsohn, P. M., \& Seeley, J. R. (1995). Symptoms versus a diagnosis of depression: Differences in psychosocial functioning. Journal of Consulting and Clinical Psychology, 63, 90-100.

Hammen, C. L. (1991a). Depression runs in families: The social context of risk and resilience in children of depressed mothers. New York: Springer-Verlag.

Hammen, C. (1991b). The generation of stress in the course of unipolar depression. Journal of Abnormal Psychology, 100, 555-561.

Hammen, C. (2002). The context of stress in families of children with depressed parents. In S. Goodman \& I. Gotlib (Eds.), Children of depressed parents: Mechanisms of risk and implications for treatment (pp. 175-199). Washington, DC: American Psychological Association.

Hammen, C., Adrian, C., Gordon, D., Burge, D., Jaenicke, C., \& Hiroto, D. (1987). Children of depressed mothers: Maternal strain and symptom predictors of dysfunction. Journal of Abnormal Psychology, 96, 190198.

Hammen, C., \& Brennan, P. (2001). Depressed adolescents of depressed and nondepressed mothers: Tests of an interpersonal impairment hypothesis. Journal of Consulting and Clinical Psychology, 69, 284-294.

Hammen, C., \& Brennan, P. (2002). Interpersonal dysfunction in depressed women: Impairments independent of depressive symptoms. Journal of Affective Disorders, 72, 145-156.

Hammen, C., Burge, D., \& Stansbury, K. (1990). Relationship of mother and child variables to child outcomes in a high-risk sample: A causal modeling analysis. Developmental Psychology, 26, 24-30.

Harter, S. (1988). Developmental and dynamic changes in the nature of the self-concept: Implications for child psychotherapy. In S. R. Shirk (Ed.), Cognitive development and child psychotherapy (pp. xv-344). New York: Plenum Press.

Hays, R. D., Wells, K. B., Sherbourne, C. D., Rogers, W., \& Spritzer, K. (1995). Functioning and well-being outcomes of patients with depression compared with chronic general medical illnesses. Archives of General Psychiatry, 52, 11-19.

Holmbeck, G. N. (1997). Toward terminological, conceptual, and statistical clarity in the study of mediators and moderators: Examples from the child-clinical and pediatric psychology literatures. Journal of Consulting and Clinical Psychology, 65, 599-610.

Horwath, E., Johnson, J., Klerman, G. L., \& Weissman, M. M. (1992) Depressive symptoms as relative and attributable risk factors for firstonset major depression. Archives of General Psychiatry, 49, 817-823.

Hu, L., \& Bentler, P. M. (1999). Cutoff criteria for fit indexes in covariance structure analysis: Conventional criteria versus new alternatives. Structural Equation Modeling, 6, 1-55.

Hu, L., Bentler, P. M., \& Kano, Y. (1992). Can test statistics in covariance structure analysis be trusted? Psychological Bulletin, 112, 351-362.

Jamshidian, M., \& Bentler, P. M. (1999). ML estimation of mean and covariance structure with missing data using complete data routines. Journal of Educational and Behavioral Statistics, 24, 21-41.

Keeping, J. D., Najman, J. M., Morrison, J., Western, J. S., Andersen, M. J., \& Williams, G. M. (1989). A prospective longitudinal study of social, psychological, and obstetrical factors in pregnancy: Response rates and demographic characteristics of the 8,556 respondents. British Journal of Obstetrics and Gynaecology, 96, 289-297.

Kline, R. B. (1998). Principles and practice of structural equation modeling. New York: Guilford Press.

Lewinsohn, P. M., Rohde, P., Klein, D. M., \& Seeley, J. R. (1999). Natural course of adolescent major depressive disorder: I. Continuity into young adulthood. Journal of the American Academy of Child and Adolescent Psychiatry, 38, 56-63

Lovejoy, C. M., Graczyk, P. A., O’Hare, E., \& Neuman, G. (2000). Maternal depression and parenting behavior: A meta-analytic review. Clinical Psychology Review, 20, 561-592.

Nomura, Y., Wickramaratne, P., Warner, V., Mufson, L., \& Weissman, M. 
(2002). Family discord, parental depression, and psychopathology in offspring: Ten-year follow-up. Journal of the American Academy of Child and Adolescent Psychiatry, 41, 402-409.

Orvaschel, H. (1995). Schedule for Affective Disorder and Schizophrenia for School-Age Children-Epidemiologic version-5. Ft. Lauderdale, FL: Nova Southeastern University, Center for Psychological Studies.

Orvaschel, H., Puig-Antich, J., Chambers, W., Tabrizi, M., \& Johnson, R. (1982). Retrospective assessment of prepubertal major depression with the Kiddie-SADS-E. Journal of the American Academy of Child and Adolescent Psychiatry, 21, 392-397.

Pine, D. S., Cohen, P., Gurley, D., Brook, J. S., \& Ma, Y. (1998). The risk for early-adulthood anxiety and depressive disorders in adolescents with anxiety and depressive disorders. Archives of General Psychiatry, 55, $56-64$.

Rao, U., Hammen, C., \& Daley, S. (1999). Continuity of depression during the transition to adulthood: A 5-year longitudinal study of young women. Journal of the American Academy of Child and Adolescent Psychiatry, 38, 908-915.

Satorra, A., \& Bentler, P. M. (1988, August). Scaling corrections for chi-square statistics in covariance structure analysis. Paper presented at the Proceedings of the American Statistical Association, Alexandria, VA.

Schludermann, S., \& Schludermann, E. (1988). Shortened Child Report of Parent Behavior Inventory (CRPBI-30): Schludermann revision. Unpublished manuscript, University of Manitoba, Winnipeg, Manitoba, Canada.
Seifer, R., Sameroff, A., Dickstein, S., Keitner, G., Miller, I., Rasmussen, S., \& Hayden, L. (1996). Parental psychopathology, multiple contextual risks, and one-year outcomes in children. Journal of Clinical Child Psychology, 25, 423-435.

Ullman, J. B. (1996). Structural equation modeling. In B. G. Tabachnick \& L. S. Fidell (Eds.), Using multivariate statistics (3rd ed., pp. 709-811). New York: Harper Collins.

Wallace, J., Schneider, T., \& McGuffin, P. (2002). Genetics of depression. In I. Gotlib \& C. Hammen (Eds.), Handbook of depression (pp. 169 191). New York: Guilford Press.

Warner, V., Mufson, L., \& Weissman, M. M. (1995). Offspring at high and low risk for depression and anxiety: Mechanisms of psychiatric disorder. Journal of the American Academy of Child and Adolescent Psychiatry, 34, 786-797.

Weissman, M. M., Wolk, S., Wickramaratne, P., Goldstein, R., Adams, P., Greenwald, S., et al. (1999). Children with prepubertal-onset major depressive disorder and anxiety grown up. Archives of General Psychiatry, 56, 794-801.

Yuan, K. H., \& Bentler, P. M. (2000). Three likelihood-based methods for mean and covariance structure analysis with nonnormal missing data. Sociological Methods, 30, 165-200.

Received November 5, 2002 Revision received August 12, 2003 Accepted August 22, 2003

\section{E-Mail Notification of Your Latest Issue Online!}

Would you like to know when the next issue of your favorite APA journal will be available online? This service is now available to you. Sign up at http://watson.apa.org/ notify/ and you will be notified by e-mail when issues of interest to you become available! 